\title{
Refractory atrioventricular nodal re-entrant tachycardia: A rare manifestation of acute massive pulmonary embolism
}

\author{
Jignesh Patel $M D^{1}$, Jinu John $M D^{2}$, Sameer Chadha $M D^{2}$, Manfred Moskovits MD², \\ Felix Yang $M D^{2}$, Gerald Hollander $M^{2}$, Jacob Shani MD²
}

\begin{abstract}
J Patel, J John, S Chadha, et al. Refractory atrioventricular nodal re-entrant tachycardia: A rare manifestation of acute massive pulmonary embolism. Curr Res Cardiol 2015;2(1):43-44.
\end{abstract}

With a wide array of manifestations ranging from mild dyspnea to fatal cardiogenic shock, acute pulmonary embolism (PE) remains an enigmatic clinical entity. Vivid electrocardiography (ECG) patterns portrayed in different clinical instances of acute PE have always remained an interesting, yet debatable subject since the classic S1Q3T3 pattern on ECG secondary to PE was first described in 1935. Atrial fibrillation, atrial flutter and atrial tachycardia

Dulmonary embolism (PE) has always been a challenging diagnosis to establish due to its variable and nonspecific presentation. Despite advances in diagnostic and therapeutic modalities, PE accounts for 300,000 deaths annually in the United States, with the past 10 years witnessing a surprising uptrend in the incidence of PE hospitalization from 129 to 302 per 100,000 person-years $(1,2)$. Hemodynamic statusbased risk stratification of PE into massive, submassive and low-risk categories has helped to streamline therapeutic decision making (3). However, new-onset cardiac arrhythmia consequent to PE can also adversely influence hemodynamic characteristics, delaying timely recognition of PE and confounding the aforementioned risk stratification. Clinicians should remain vigilant to recognize PE in the backdrop of new-onset rhythm disturbances in appropriate settings.

\section{CASE PRESENTATION}

On the morning of admission, an 89-year-old Caucasian woman with a medical history of dementia, hypertension and chronic obstructive pulmonary disease was noted to experience sudden-onset dyspnea (oxygen desaturation to $80 \%$ on room air), hypotension and tachycardia at the nursing and rehabilitation centre; emergency medical services was called and she was intubated in the field. According to her history, she had not reported chest pain, diaphoresis, nausea or vomiting. She had been admitted to the rehabilitation centre one month previously, following conservative management at another hospital for blunt sternal trauma secondary to a fall of unknown nature at her home. She had been mostly bedridden for the past month and was taking metoprolol $25 \mathrm{mg}$ orally every $12 \mathrm{~h}$, budesonide $0.5 \mathrm{mg}$ nebulization every $12 \mathrm{~h}$, famotidine $20 \mathrm{mg}$ orally every $12 \mathrm{~h}$ and magestrol $400 \mathrm{mg}$ orally once daily. She was brought to the emergency department (ED) having already been intubated by emergency medical services. On admission to the ED, her heart rate was regular but tachycardic ( 170 beats/min), with a respiratory rate of 34 breaths/min, an oxygen saturation of $96 \%$ on $100 \% \mathrm{FiO}_{2}$ on a mechanical ventilator, blood pressure (BP) of $73 / 60 \mathrm{mmHg}$ and body temperature $98.8^{\circ} \mathrm{C}$; she was drowsy and completely unaware of her surroundings. A respiratory system examination revealed bilateral equal air entry without any adventitious sounds, and cardiovascular examination was notable for tachycardia.

\begin{abstract}
are commonly described supraventricular tachycardias in association with $\mathrm{PE}$; however, there is scant description of PE in the literature presenting as atrioventricular nodal re-entrant tachycardia (AVNRT). The present report describes the clinical course of an elderly woman presenting with refractory typical AVNRT who was subsequently found to have bilateral multilobar acute PE. To the authors' knowledge, the present report is the first to describe a case involving acute PE presenting as hemodynamically unstable AVNRT requiring cardioversion.
\end{abstract}

Key Words: AVNRT; Pulmonary embolism

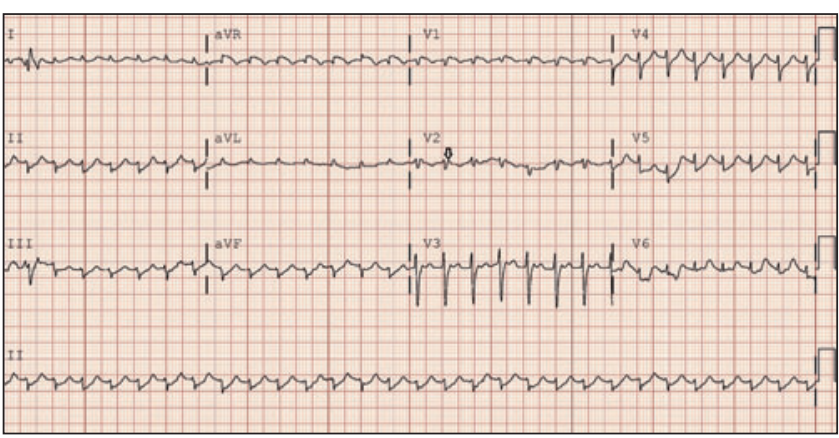

Figure 1) Electrocardiogram on admission showing narrow complex tachycardia (174 beats/min). The arrow indicates the characteristic pseudo $R$ prime pattern apparent in precordial leads in atrioventricular nodal re-entrant tachycardia

A 12-lead electrocardiogram obtained on admission (Figure 1) revealed typical AVNRT, with a heart rate of 174 beats/min and low voltage in the limb leads. Because she was hemodynamically unstable, synchronized cardioversion was attempted twice with $150 \mathrm{~J}$ followed by $200 \mathrm{~J}$, which converted her to sinus rhythm only transiently, with improved BP. She again developed AVNRT, and $6 \mathrm{mg}$ intravenous adenosine followed by $12 \mathrm{mg}$ intravenously was attempted, which transiently normalized the arrhythmia; however, she again reverted into AVNRT (Figure 2). She received a bolus with $2 \mathrm{~L}$ normal saline in the first hour. After her BP improved, she received $10 \mathrm{mg}$ of intravenous diltiazem and consequently maintained sinus rhythm. Cardiac troponin and creatine kinase-MB were mildly elevated $(0.43 \mathrm{ng} / \mathrm{mL}$ and $12.8 \mathrm{ng} / \mathrm{mL}$, respectively) and her B-type natriuretic peptide level was $324 \mathrm{ng} / \mathrm{L}$. A chest $\mathrm{x}$-ray revealed unilateral pulmonary oligemia over the right lung field (Figure 3) and $\mathrm{ABG}$ showed a significantly elevated alveolar-arterial gradient. A bedside echocardiogram (Video 1) showed right ventricular dysfunction with free wall hypokinesis sparing the apex (McConnell's sign) and normal left ventricular ejection fraction of $60 \%$ to $65 \%$. At this point, with a high pretest probability for acute PE, she

Department of Internal Medicine; Department of Cardiology, Maimonides Medical Center, Brooklyn, New York, USA

Correspondence: Dr Jinu John, Department of Cardiology, Maimonides Medical Center, 4802 Tenth Avenue, Brooklyn, New York 11219, USA.

Telephone 718-283-7489, fax 718-283-8546,e-mail doctorjacob@gmail.com, jjohn3@maimonidesmed.org 


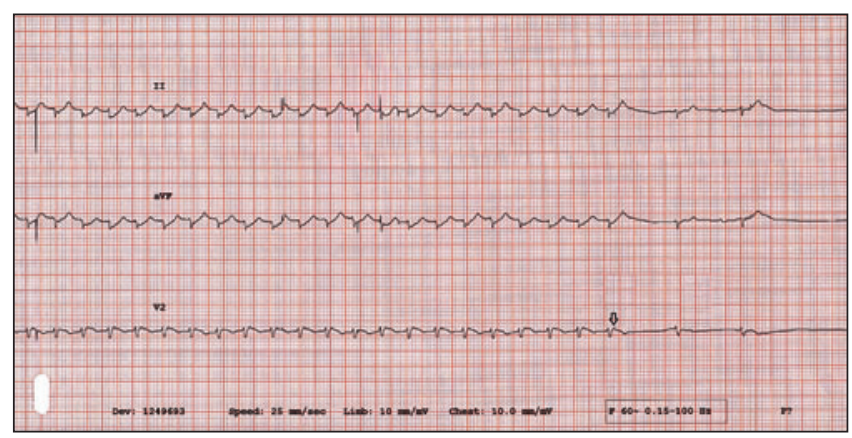

Figure 2) Rhythm strip at the time of administration of adenosine showing termination of the tachycardia. Also note the disappearance of pseudo $R$ prime pattern (arrow) once the tachycardia terminates

received an intravenous heparin bolus followed by intravenous heparin drip and underwent computed tomography pulmonary angiography, which revealed high-volume multilobar pulmonary emboli involving right upper, middle and lower lobe and left upper lobe and lingula (Video 2). She was transferred to the medical intensive care unit. Venous Doppler ultrasonography of her lower extremities showed acute partial thrombosis in the left common femoral vein.

She was switched to rivaroxaban $15 \mathrm{mg}$ through a feeding tube every $12 \mathrm{~h}$ beginning the day after her admission. During hospitalization, she developed left lower lobe consolidation and was treated with intravenous antibiotics. She was extubated on hospitalization day 6, maintained sinus rhythm throughout and remained stable during the remainder of her treatment.

\section{DISCUSSION}

With 900,000 incident or recurrent annual cases and a high average fatality rate of $15 \%$, PE remains the third most common cardiovascular diagnosis in United States $(4,5)$. Given the frequent disguised clinical presentation of $\mathrm{PE}$, incorporation of basic diagnostic tools, such as electrocardiography, chest $\mathrm{x}$-rays and two-dimensional echocardiography with clinical profile, is essential for the early identification of this 'great masquerader'. Our patient had the Westermark sign on chest $\mathrm{x}$-ray (focal pulmonary oligemia), AVNRT on electrocardiography and McConnell's sign on two-dimensional echocardiography with pertinent clinical background of being bedridden due to generalized debilitation.

Preexisting cardiovascular status of the patient, in conjunction with different cardiovascular responses elicited by $\mathrm{PE}$, results in the numerous electrocardiography patterns previously described in the literature. The markedly variable frequency of these electrocardiographic changes reported in various case series impairs sensitivity and specificity of electrocardiography as a diagnostic tool for PE $(6,7)$. Nevertheless, new-onset atrial arrhythmias, new right bundlebranch block (complete or incomplete), Qr pattern in V1, S1Q3T3, negative $\mathrm{T}$ waves in $\mathrm{V} 1$ through $\mathrm{V} 4$, and ST-segment shift over V1 through $\mathrm{V} 4$ have been shown to correlate with poorer short-term prognosis in acute PE (8).

Hemodynamic response to PE is determined by the size of the embolus, the patient's underlying cardiopulmonary status and compensatory neurohumoral adaptations (9). However, when PE manifests as new-onset cardiac arrhythmia, the rhythm disturbance is not only usually attributed as a primary culprit for the patient's altered hemodynamics, but may also delay identifying PE as primary insult. PE presenting as typical slow-fast AVNRT is rarely described in literature. In our patient, we believe that multiple atrial premature complexes due to the underlying PE persisted in triggering AVNRT refractory to standard treatment including adenosine and synchronized DC cardioversion. By definition, our patient experienced a massive PE; however, considering her advanced age, overall poor functional status, confounding effects of refractory AVNRT on

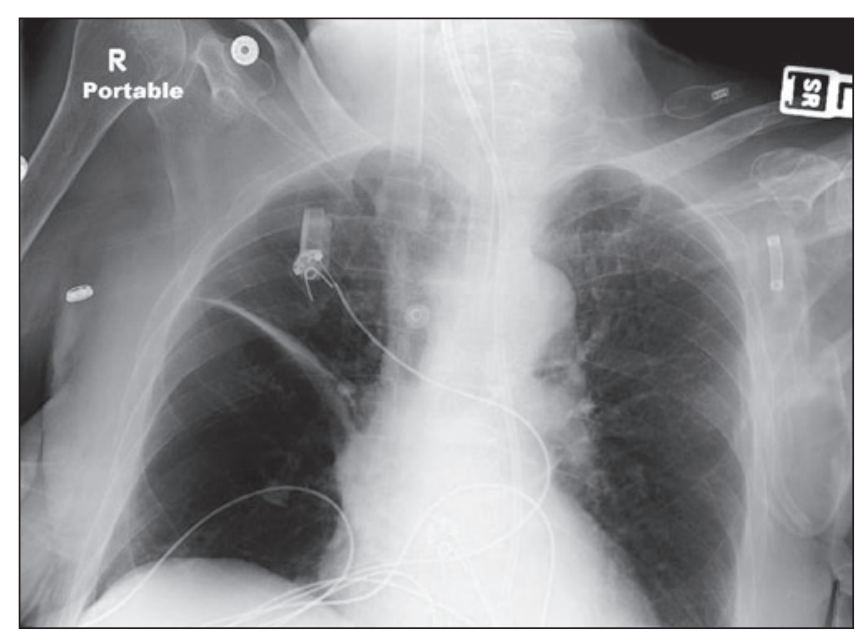

Figure 3) Chest $x$-ray showing focal oligemia in lower right lung field (Westermark sign)

hemodynamics and improvement in hemodynamic status, thrombolysis or embolectomy were not considered and she was treated with heparin infusion and, subsequently, with rivaroxaban, with favourable outcomes.

We reported an often unrecognized causal relationship between PE and typical AVNRT refractory to standard treatment, timely identification of which can be lifesaving. Complex interplay of new-onset cardiac arrhythmia with underlying PE and its influence on the patient's hemodynamics should be taken into consideration before selecting contemporary management strategy for PE based on definition of massive versus submassive PE.

DISCLOSURES: The authors have no financial disclosures or conflicts of intertest to declare.

ACKNOWLEDGEMENTS: The authors thank Svetlana Khentov, a sonographer at Maimonides Medical Center.

\section{REFERENCES}

1. McCord J, Borzak S. Multifocal atrial tachycardia.

Chest 1998;113:203-09.

2. Minges KE, Bikdeli B, Wang Y, et al. National trends in pulmonary embolism hospitalization rates and outcomes for Medicare beneficiaries, 1999-2010. J Am Coll Cardiol 2013;61.

3. Tapson VF. Medical progress: Acute pulmonary embolism. N Engl J Med 2008;358:1037-52.

4. Goldhaber SZ Visani L, De Rosa M. Acute pulmonary embolism: Clinical outcomes in the International Cooperative Pulmonary Embolism Registry (ICOPER): Lancet 1999;353:1386-9

5. Roger VL, Go AS, Lloyd-Jones DM, et al. American Heart Association Statistics Committee and Stroke Statistics Subcommittee. Heart disease and stroke statistics - 2012 update: A report from the American Heart Association. Circulation 2012;125:e2-e220

6. Rodger M, Makropoulos D, Turek M, et al. Diagnostic value of the electrocardiogram in suspected pulmonary embolism. Am J Cardiol 2000;86:807-9.

7. Stein PD, Terrin ML, Hales CA, et al. Clinical, laboratory, roentgenographic, and electrocardiographic findings in patients with acute pulmonary embolism and no pre-existing cardiac or pulmonary disease. Chest 1991;100:598-603.

8. Jaff MR, McMurtry MS, Archer SL, et al. Management of massive and submassive pulmonary embolism, iliofemoral deep vein thrombosis, and chronic thromboembolic pulmonary hypertension: A scientific statement from the American Heart Association. Circulation 2011;123:1788-830

9. Piazza G, Goldhaber SZ. Acute pulmonary embolism, part I: Epidemiology and diagnosis. Circulation 2006;114:e28-e32. 